\title{
NONLINEAR SITE RESPONSE ANALYSIS BY COUPLING SCALED BOUNDARY FINITE ELEMENT METHOD AND FINITE ELEMENT METHOD
}

\author{
Ali BARGHI KHERZELOO and Nader HATAF * \\ Faculty of Engineering 1, Department of Civil and Environmental Engineering, Shiraz University, Zand Avenue, Shiraz, Iran \\ *Corresponding author's e-mail: nhataf@shirazu.ac.ir
}

\section{ARTICLE INFO}

\section{Article history:}

Received 25 May 2020

Accepted 31 August 2020

Available online 19 September 2020

\section{Keywords:}

Nonlinear analysis

Site response

SBFEM

OpenSees

Coupling method

\begin{abstract}
This paper presented 2D numerical linear and nonlinear site response analyses based on the scaled boundary finite-element method (SBFEM) and compared their results with those of the DEEPSOIL software. In linear time-domain analysis, the seismic boundary traction was applied to lines in the near-field with the same vertical coordinates using seismic time history load. The far-field was modeled utilizing an improved continued-fraction-based high-order transmitting boundary. The constitutive relationship of the boundary was determined utilizing the SBFEM equation in the dynamic stiffness model. It was shown that the results of the SBFEM had a good agreement with those obtained from the DEEPSOIL software. The results of spectral acceleration demonstrated period lengthening. The nonlinear site responses were analyzed using both the DEEPSOIL software and the coupling of SBFEM/FEM. The one-dimensional nonlinear site response was analyzed using the tools in the DEEPSOIL software including the strength correction, pressure-dependent modulus reduction, and the damping ratio curve of sand. In the nonlinear-coupled analysis, the bounded domain was also modeled in OpenSees using a pressuredependent multi-yield plasticity soil model. The comparison of the results demonstrated the accuracy of the nonlinear analysis using the coupled SBFEM/FEM. The coupling method underestimated spectral acceleration in low periods compared with the DEEPSOIL software. The absolute residual was also obtained less than 0.2 .
\end{abstract}

\section{INTRODUCTION}

The soil condition has a great influence on earthquake ground motion. This effect is known as the 'site response'. Amplification factors (AF) are utilized to demonstrate the site effect. AF is defined as the ratio of Fourier spectral ordinates of motion recorded on top of the soil layer to those recorded on the rock underneath the soil. Several studies have been conducted to assess this factor (Harmsen, 1997; Field and Petersen, 2000; Joyner and Boore, 2000). In 2005, Choi and Stewart introduced a nonlinear site amplification relationship as a function of $\mathrm{V}_{\mathrm{s}, 30}$ which is the soil shear velocity at a depth of 30 meters. They explained that the nonlinearity effect was high for $\mathrm{V}_{\mathrm{s}, 30}$ $<180 \mathrm{~m} / \mathrm{s}$. This effect was reduced to a small value in the high shear velocity of soil $\left(\mathrm{V}_{\mathrm{s}, 30}>300 \mathrm{~m} / \mathrm{s}\right)$.

In addition to the typical AF, the linear (L), equivalent linear (EQL), and nonlinear (NL) methods can also be used for 1D modeling of the site response. In 1969, Seed and Idriss introduced an equivalent linear method using a linear spring and dashpot to model shear modulus and damping, respectively.

In the 1D non-linear analysis, the vertical soil profile is discretized into $\mathrm{n}$ layers using a multi-degreeof-freedom lumped parameter model. Another method utilizes the finite element method to solve a dynamic equilibrium equation at each time step using the Newmark integration method. Figure 1 illustrates the lumped mass parameter model.

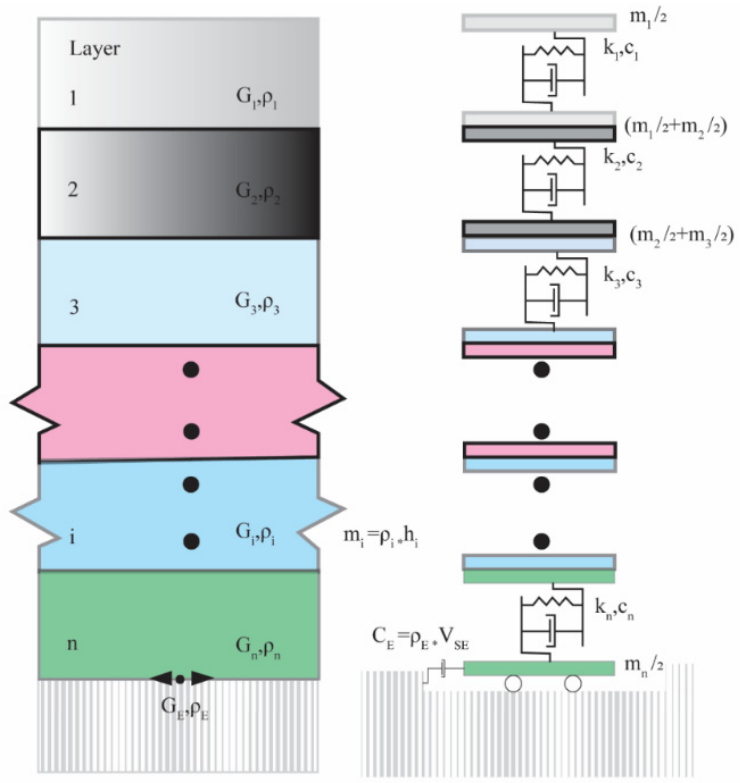

Fig. 1 Lumped mass modeling. 
Park and Hashash formulated soil damping for nonlinear response analysis in 2004. In 2009, Philips and Hashash proposed constitutive models for modulus reduction and soil damping. They introduced a viscous damping parameter in NL analysis to overcome overdamping at high frequencies.

Elgamal et al. in 2001 and Tsai and Hashash in 2009 used downhole array data to obtain a proper dynamic soil model for seismic site response analysis by employing a novel inverse analysis framework. In 2010, Hashash et al. introduced some NL analysis methods and proposed a procedure to define a proper seismic constitutive model of soil. Kwok et al. recommended a guideline for code usage and parameter selection in nonlinear site response analysis in 2008. In their study, the Rayleigh damping procedure and the target backbone were determined. In 2019, Afacan et al. studied the site response analysis of soft soil using centrifuge modeling. In 2015, Hashash et al. demonstrated the reliability of NL site response analysis compared with the centrifuge test data.

In 2013, Yee analyzed the NL and EQL site responses by using a modified soil model in DEEPSOIL to overcome the strain localization problem. In 2016, Pruiksma compared the differences among the results of the EQL, NL, and linear elastic models. The NL method led to a lower peak ground acceleration (PGA) at high acceleration levels and a small difference in the short spectral period. In 2015, Kaklamanos et al. used L, NL, and EQL models to analyze KiK-net sites and showed that NL and EQL models had more accurate results. Kim et al. investigated the relative difference between NL and EQL site response analysis methods in 2016. In 2014, Tsai and Chen compared different analysis methods and demonstrated that the NL method was better than the other approaches in response analysis. In 2006, Arsalan and Siyahi compared the NL and L site response analysis methods using the SHAKE software and summarized well-known site response analysis methods.

In 2018, Regnier et al. selected 5 sites and 19 input motions as international benchmarks. The soil properties were obtained using experimental and field tests. The 1-dimensional nonlinear total stress analysis was performed and the uncertainty of the responses was reported to range from 0.05 to 0.25 in the log scale. The NL site response has been analyzed in various studies (Rong et al., 2016; Mirshekari and Ghayoomi, 2015; Lopez et al., 2007; Kwok et al., 2007; Seyhan and Stewart, 2014; Hashash and Moon, 2011).

$2 \mathrm{D}$ site response analysis has a better estimation of the characteristics of surface ground motion in case the site has a steep valley or canyon. The $1 \mathrm{D}$ site response analysis should be replaced by the $2 \mathrm{D}$ site response analysis in a place that has inclined soil layers or when the soil-structure interaction needs to be studied. In the $2 \mathrm{D}$ site response analysis, the efficient boundary conditions have a significant effect on the results.

The simulations in this paper were performed using the scaled boundary finite element method (SBFEM) for modeling time history analysis. The SBFEM formulations are mentioned in section 2 . The linear site response was analyzed by both DEEPSOIL and the SBFEM. The parameters of the 1-dimensional site response analysis and the constitutive model for the damping and shear modulus reduction curves were selected from previous studies. The traction of the seismic load was obtained based on the studies of Joyner and Chen (1975) and Lysmer and Kuhleymer (1969). This dynamic traction was applied as a time history of traction in the SBFEM. The nonlinear response analysis was performed using DEEPSOIL and the coupling of SBFEM and OpenSees. The results of both L and NL analyses were presented and the differences were highlighted.

\section{THE SUMMARY OF THE SBFEM}

The scaled boundary finite element method is a novel semi-analytical formulation for solving partial differential equations. This method has the advantages of both FEM and BEM without requiring a fundamental solution. This method was first developed by Song and Wolf in 1998. In 2006, Song used the eigenvalue solution and the spectral shifting technique in the SBFEM to overcome the problems caused by the sparsity of the global stiffness matrix.

In 2007, Song and Bazyar developed a Padé series solution for the frequency domain and determined the continued fraction based on the original SBFEM equations. In their study, the identity matrix was considered as an auxiliary variable. In 2012, Birk et al. developed an improved high-order transmitting boundary for time-domain analysis. They proposed the SBFEM/FEM coupling method by utilizing an improved high-order transmitting boundary. In 2017, Bazyar and Song studied the site response analysis by employing SBFEM and applying the Ricker wavelet displacement signals to a node. The results showed a good agreement between the analytical solution and the SBFEM modeling.

A comprehensive description of the derivation of the equations used in the SBFEM has been given by Wolf (2003) and Song (2018). In this method, the whole domain is discretized into many subdomains and the SBFEM equations are solved in each subdomain. Each subdomain has a scaling center as shown in Figure 2.

In a $2 \mathrm{D}$ problem, only the line elements are discretized and the equations of motion (Eq. 1) are calculated in a transformed scaled space with $\xi, \eta$, and $\zeta$ coordinates. The equation is obtained on the radial coordinate $(\xi)$ as:

$$
D^{T} \sigma+\mathrm{f}+\omega^{2} \rho \mathrm{u}=0
$$




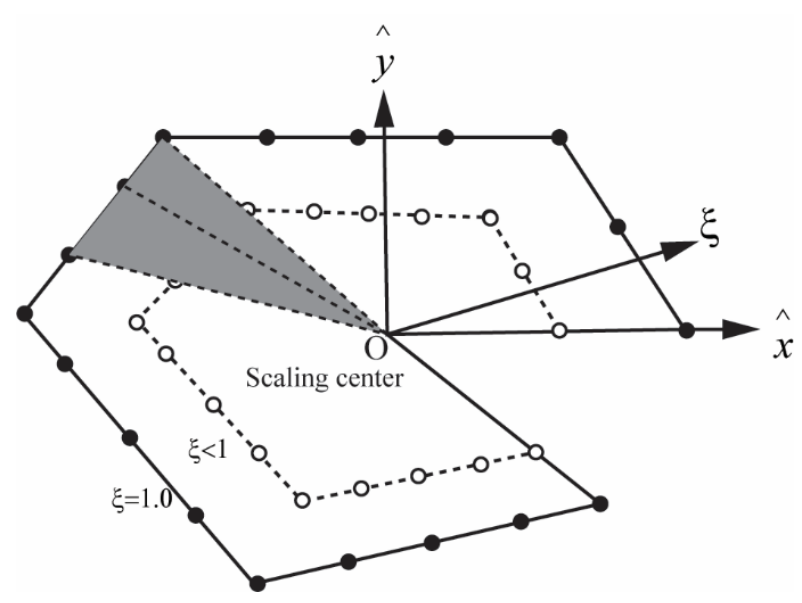

Fig. 2 The scaling center in the SBFEM.

where $\sigma$ is stress corresponding to displacement $(\mathrm{u})$, $\omega$ is the excitation frequency, and $\mathrm{f}$ is the body force. The Cartesian coordinate system for a threedimensional space is converted into scaled coordinates and all the equations are obtained in scaled coordinates as follows:

$$
\begin{aligned}
& \hat{x}(\xi, \eta, \zeta)=\xi[N(\eta, \zeta)]\{x\} \\
& \hat{y}(\xi, \eta, \zeta)=\xi[N(\eta, \zeta)]\{y\} \\
& \hat{z}(\xi, \eta, \zeta)=\xi[N(\eta, \zeta)]\{z\}
\end{aligned}
$$

where $[N(\eta, \zeta)]$ is a shape function.

The unknown variable $\{u(\xi, \eta, \zeta)\}$ is related to $\{u(\xi)\}$ as:

$$
\{u(\xi, \eta, \zeta)\}=\left[N^{u}(\eta, \zeta]\{u(\xi)\}\right.
$$

The Jacobian matrix is used to transform $(\mathrm{x}, \mathrm{y}, \mathrm{z})$ to $(\xi, \eta, \zeta)$ and then the derivation relationships for all the unknown variables are determined as:

$$
\begin{array}{r}
{\left[\begin{array}{c}
\frac{\partial}{\partial x} \\
\frac{\partial}{\partial y} \\
\frac{\partial}{\partial z}
\end{array}\right]=\frac{1}{|\mathrm{~J}|}\left(\begin{array}{ccc}
n_{1}^{\xi} & n_{1}^{\eta} & n_{1}^{\zeta} \\
n_{2}^{\xi} & n_{2}^{\eta} & n_{2}^{\zeta} \\
n_{3}^{\xi} & n_{3}^{\eta} & n_{3}^{\zeta}
\end{array}\right)\left[\begin{array}{l}
\frac{\partial}{\partial \xi} \\
\frac{\partial}{\xi} \frac{\partial}{\partial \eta} \\
\frac{1}{\xi} \frac{\partial}{\partial \zeta}
\end{array}\right]=} \\
=\frac{n^{\xi}}{|\mathrm{J}|} \frac{\partial}{\partial \xi}+\frac{1}{\xi}\left(\frac{n^{\eta}}{|\mathrm{J}|} \frac{\partial}{\partial \eta}+\frac{n^{\zeta}}{|\mathrm{J}|} \frac{\partial}{\partial \zeta}\right)
\end{array}
$$

where $n^{\xi}, n^{\eta}$, and $n^{\zeta}$ are the outward normal vectors to the surfaces.

Either a virtual work method or Galerkin's residual method is utilized in the $(\xi, \eta, \zeta)$ domain to obtain the SBFEM as follows:

$$
\begin{aligned}
& {\left[E^{0}\right] \xi^{2} u_{, \xi \xi}+\left((s-1)\left[E^{0}\right]-\left[E^{1}\right]+\left[E^{1}\right]^{T}\right) \xi u_{, \xi}} \\
& +\left((s-2)\left[E^{1}\right]^{T}-\left[E^{2}\right]\right) u+\omega^{2}\left[M^{0}\right] \xi^{2} u=0
\end{aligned}
$$

The subscripts are the partial derivatives of the unknown variables with respect to $\xi . E^{0}, E^{1}, E^{2}$, and $M^{0}$ in Eq. 5 are the coefficient matrices and are expressed as:

$$
\begin{aligned}
& {\left[E^{0}\right]=\int_{-1}^{1} \int_{-1}^{1}\left[B^{1}(\eta, \zeta)\right]^{T}\left[B^{1}(\eta, \zeta)\right]|J(\eta, \zeta)| d \eta d \zeta} \\
& {\left[E^{1}\right]=\int_{-1}^{1} \int_{-1}^{1}\left[B^{2}(\eta, \zeta)\right]^{T}\left[B^{1}(\eta, \zeta)\right]|J(\eta, \zeta)| d \eta d \zeta} \\
& {\left[E^{2}\right]=\int_{-1}^{1} \int_{-1}^{1}\left[B^{2}(\eta, \zeta)\right]^{T}\left[B^{2}(\eta, \zeta)\right]|J(\eta, \zeta)| d \eta d \zeta} \\
& {\left[M^{0}\right]=\frac{1}{c^{2}} \int_{-1}^{1} \int_{-1}^{1}[N(\eta, \zeta)]^{T}[N(\eta, \zeta)]|J(\eta, \zeta)| d \eta d \zeta}
\end{aligned}
$$

B1 and B2 matrices are:

$$
\begin{aligned}
& {\left[B^{1}\right]=\left[b^{1}\right][N]} \\
& {\left[B^{2}\right]=\left[b^{2}\right]\left[N_{, \eta}\right]+\left[b^{3}\right]\left[N_{, \zeta}\right]}
\end{aligned}
$$

For more details about deriving $\left[b^{1}\right]$ and $\left[b^{2}\right]$ refer to Birk et al. (2012). Equation 11 demonstrates the subdomain solution procedure in the original SBFEM. The boundary tractions are integrated over the elements.

$$
\{q(\xi)\}=\xi^{(s-2)}\left(\left[E^{0}\right] \xi\{u(\xi)\}_{, \xi}+\left[E^{1}\right]^{T}\{u(\xi)\}\right) .
$$

where $s$ is related to the spatial dimension of the domain. On the boundary between the unbounded domain and the bounded domain, the forcedisplacement relationship is defined as follows:

$$
\{R(\omega)\}=\left[S^{\infty}(\omega)\right]\{u(\omega)\}
$$

The SBFEM formulation for the interaction zone is determined by using Eq. 5: 


$$
\left(\left[S^{\infty}(\omega)\right]+\left[E^{1}\right]\right)\left[E^{0}\right]^{-1}\left(\left[S^{\infty}(\omega)\right]+\left[E^{1}\right]^{T}\right)-(s-2)\left[S^{\infty}(\omega)\right]-\omega\left[S^{\infty}(\omega)\right]_{, \omega}-\left[E^{2}\right]+\omega^{2}\left[M^{0}\right]=0
$$

In the load-deflection relationship in the boundary between the far-field and near-field $(\xi=1.0)$, stiffness is expressed as:

$$
\left[S^{\infty}(\omega)\right]=i \omega\left[C_{\infty}\right]+\left[K_{\infty}\right]-\left[R^{(1)}(\omega)\right]
$$

The residual term $\left[R^{(1)}(\omega)\right]$ is defined as:

$$
\left[R^{(1)}(\omega)\right]=\left[X^{(i)}\right]\left[Y^{(i)}(\omega)\right]^{-1}\left[X^{(i)}\right]^{T}
$$

Birk et al. (2012) have given more details about the residual term. In 2008, Bazyar and Song obtained the continued fraction transmitting boundary formulation and Birk et al. expanded it to a high order in 2012. The motion equation of the SBFEM is described as:

$$
\{R(\omega)\}=\left[S^{\infty}(\omega)\right]\{u(\omega)\}=\left(i \omega\left[C_{\infty}\right]+\left[K_{\infty}\right]\right)\{u(\omega)\}-\left[X^{(1)}\right]\left\{u^{1}(\omega)\right\}
$$

The auxiliary variable $\left\{u^{1}(\omega)\right\}$ is defined as:

$$
\left\{u^{1}(\omega)\right\}=\left[Y^{(1)}(\omega)\right]^{-1}\left[X^{(1)}\right]^{T}\{u(\omega)\}
$$

All auxiliary variables can be defined recursively as:

$$
\left\{u^{i}(\omega)\right\}=\left[X^{(i+1)}\right]^{-T}\left[Y^{(i+1)}(\omega)\right]^{T}\left\{u^{(i+1)}(\omega)\right\}
$$

where $\left\{u^{M+1}(\omega)\right\}=0.0$

In this research, a recursive solution was obtained for computing the auxiliary variables:

$$
\begin{aligned}
& \left\{u^{i}(\omega)\right\}=\left[t^{i}\right]\left\{u^{i-1}(\omega)\right\} \quad i=1: M \\
& {\left[t^{M}\right]=\left(Y_{0}^{M}+i \omega Y_{1}^{M}\right)^{-1}\left[X^{M}\right]^{T}} \\
& {\left[t^{M-n}\right]=\left(Y_{0}^{M-n}+i \omega Y_{1}^{M-n}-\left\{X^{M-n+1}\right\}\left[t^{M-n+1}\right]\right)^{-1}\left[X^{M-n}\right]^{T}, n=1: M-1}
\end{aligned}
$$

When all the auxiliary variables are defined, the force-displacement relationship is expressed as:

$$
\left(\left[K_{u}\right]\{Z(t)\}+\left[C_{u}\right]\{\dot{Z}(t)\}=\{f(t)\}\right.
$$

where $\left[K_{u}\right]$ and $\left[C_{u}\right]$ are frequency independent matrices and $\{Z(t)\}$ is a variable matrix. All these matrices and the external excitation are defined as:

$$
\left[K_{u}\right]=\left[\begin{array}{ccccc}
{\left[K_{\infty}\right]} & -\left[X^{(1)}\right] & 0 & 0 & 0 \\
-\left[X^{(1)}\right]^{T} & {\left[Y_{0}^{(1)}\right]} & -\left[X^{(2)}\right] & 0 & 0 \\
0 & -\left[X^{(2)}\right]^{T} & {\left[Y_{0}^{(2)}\right]} & 0 & 0 \\
\vdots & \vdots & \ddots & -\left[X^{(M-1)}\right] & 0 \\
0 & 0 & -\left[X^{(M-1)}\right]^{T} & {\left[Y_{0}^{(M-1)}\right]} & {\left[X^{(M)}\right]} \\
0 & 0 & 0 & -\left[X^{(M)}\right]^{T} & {\left[Y_{0}^{(M)}\right]}
\end{array}\right]
$$

$$
\left[C_{u}\right]=\operatorname{diag}\left(\left[C_{\infty}\right],\left[Y_{1}^{(1)}\right],\left[Y_{1}^{(2)}\right], \cdots,\left[Y_{1}^{(M-1)}\right],\left[Y_{1}^{(M)}\right]\right)
$$

$$
\{Z(t)\}=\left\{\begin{array}{c}
u \\
u^{(1)} \\
u^{(2)} \\
\vdots \\
u^{(M-1)} \\
u^{(M)}
\end{array}\right\}\{f(t)\}=\left\{\begin{array}{c}
\{R(t)\} \\
0 \\
0 \\
\vdots \\
0 \\
0
\end{array}\right\}
$$



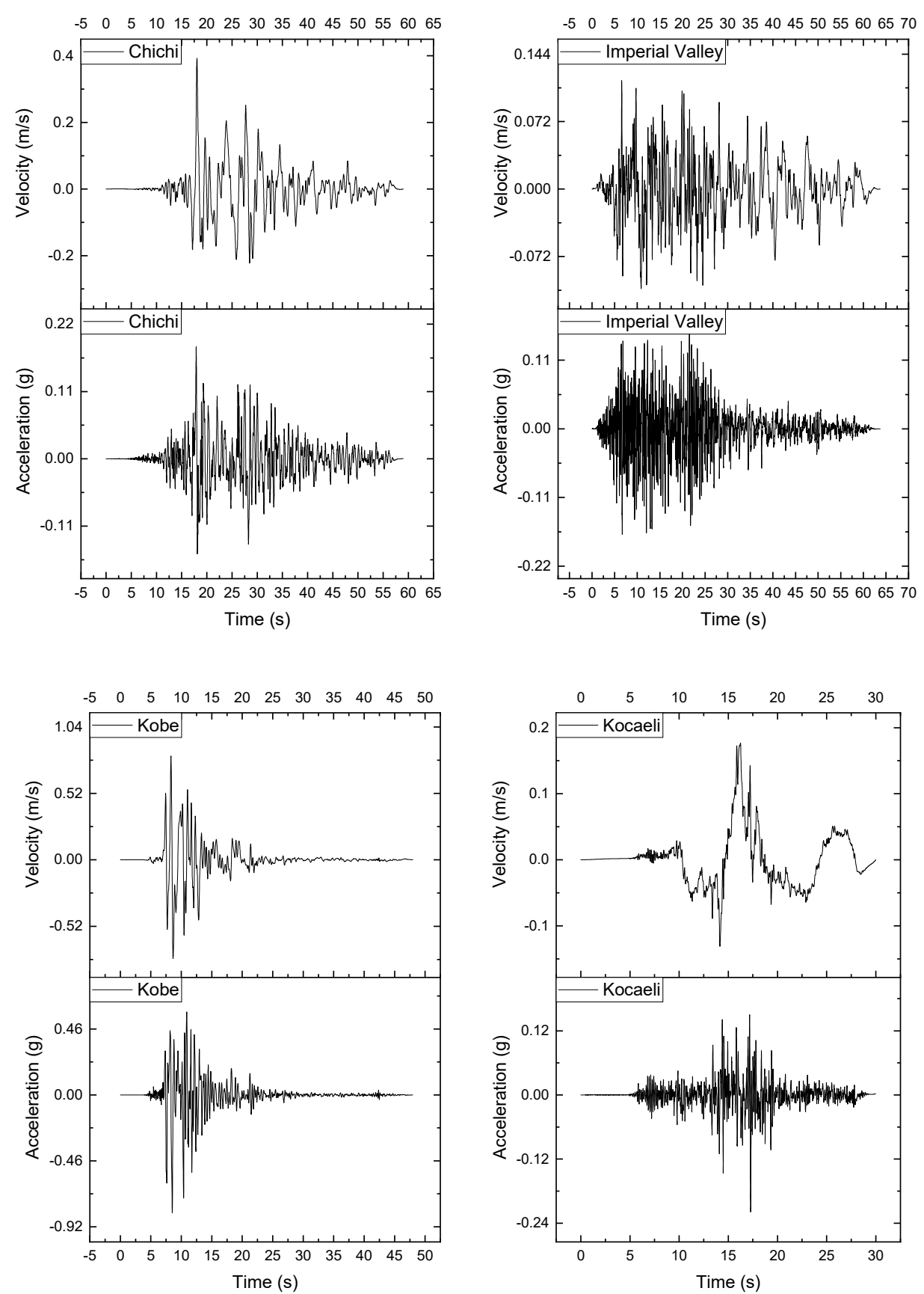

Fig. 3 Ground input motions.

3. THE NUMERICAL MODEL

L and NL models were established using both DEEPSOIL (version 7) and SBFEM/FEM methods.

\subsection{THE INPUT MOTION}

Four shaking events were applied to the boundaries in the DEEPSOIL and SBFEM models in the horizontal direction. These time histories consisted of Chichi, Imperial Valley, Kobe, and Kocaeli earthquake records (Ancheta et al., 2014). The input velocity and acceleration time history of earthquake ground motions used in this study are presented in Figure 3.

The frequency content parameters of each shaking event are tabulated in Table 1. In the linear analysis, the ground motion records were applied in an elastic half-space. Within record was applied to the bedrock half-space based on the study of Kwok et al. (2008). The bedrock was modeled by an elastic halfspace by considering the specified $\mathrm{V}_{\mathrm{s}}$ and the unit weight. All the bedrock characteristics for L and NL analyses are tabulated in Table 2. 
Table 1 Frequency content parameters.

\begin{tabular}{lcccc}
\hline Record name & $\begin{array}{c}\text { Predominant } \\
\text { period }(\mathrm{s})\end{array}$ & $\begin{array}{c}\text { Mean Period } \\
(\mathrm{s})\end{array}$ & $\begin{array}{c}\mathrm{v}_{\max } / \mathrm{a}_{\max } \\
(\mathrm{s})\end{array}$ & $\begin{array}{c}\text { Arias intensity } \\
(\mathrm{m} / \mathrm{s})\end{array}$ \\
\hline Kobe & 0.34 & 0.646 & 0.1 & 8.391 \\
Chichi & 0.54 & 0.987 & 0.219 & 0.96 \\
Imperial Valley & 0.32 & 0.389 & 0.070 & 1.222 \\
Kocaeli & 0.16 & 0.306 & 0.082 & 0.290 \\
\hline
\end{tabular}

Table 2 Bedrock specifications.

\begin{tabular}{lccc}
\hline Modeling type & $\mathrm{V}_{\mathrm{s}}(\mathrm{m} / \mathrm{s})$ & Unit weight $\left(\mathrm{kN} / \mathrm{m}^{3}\right)$ & Damping ratio (\%) \\
\hline Linear DEEPSOIL & 750 & 22 & 2 \\
Linear SBFEM & & 22 & 2 \\
$\begin{array}{l}\text { Nonlinear DEEPSOIL } \\
\text { SBFEM/FEM }\end{array}$ & 1800 & 22 \\
\hline
\end{tabular}

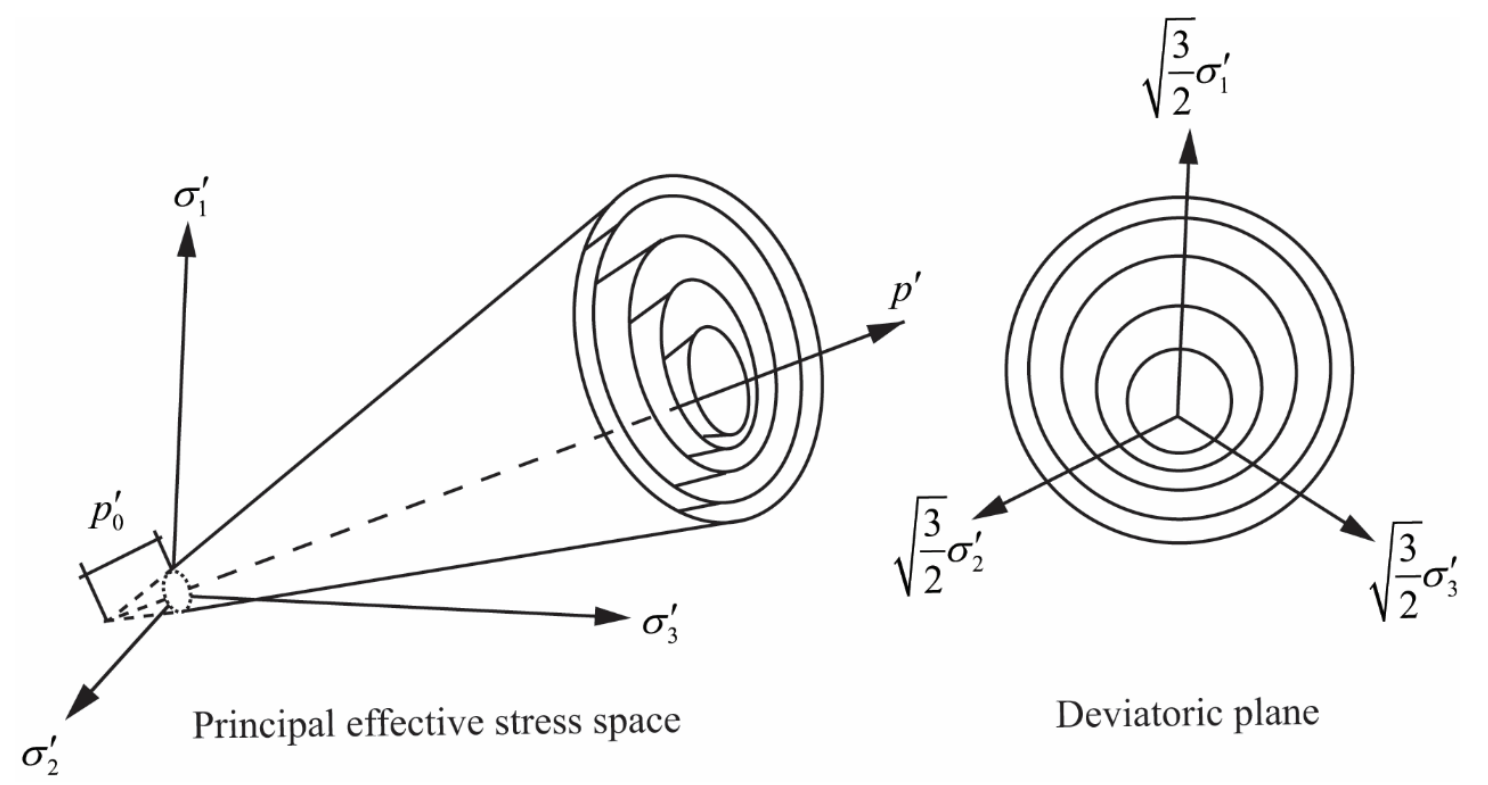

Fig. 4 The nested multi-yield surface model.

In the NL coupled SBFEM/FEM model, the velocity time history was used to apply force on the bounded domain based on the study of Kuhleymer (1969). This method was used to compute traction in the SBFEM modeling.

\subsection{THE NONLINEAR CONSTITUTIVE SOIL MODEL}

For the 1D nonlinear site response analysis, the General Quadratic/Hyperbolic (GQ/H) model with no Masing reloading unloading formulation was selected in DEEPSOIL. This software is capable of modeling the nonlinear behavior and shear strength of soil. Darandeli's target curve (2001) was used as a reference for fitting the constitutive model curve. The frequency-independent damping model was utilized in this modeling. The implicit Newmark integration method was selected for the time-domain analysis. In nonlinear coupled SBFEM/FEM modeling, the soil was modeled using a pressure- dependent multi-yield surface (PDMY) constitutive model in the OpenSees software. This constitutive model uses two stages to model the soil behavior. In stage zero, the behavior of the material is linear elastic which is suitable for gravity modeling and in-situ stress analysis. In stage one, the plastic behavior of the soil is modeled using the nested yield surface with non-associated flow rule concept.

The yield surface is illustrated in Figure 4 and all the constitutive parameters used in this study are tabulated in Table 3.

For the consistency of the coupled SBFEM model with the FEM model, the quadratic element type was selected in both FEM and SBFEM models.

\subsection{DAMPING MODELING}

In 1945, Rayleigh and Lindsay developed a damping matrix from the sum of two matrices proportional to mass and stiffness as shown in Eq. 24. 
Table 3 Multi-yield surface parameters and descriptions (Mazzoni et al., 2006).

\begin{tabular}{|c|c|}
\hline Parameter & Description \\
\hline Rho & Saturated soil mass density. \\
\hline refShearModul & $\begin{array}{l}\text { Reference low-strain shear modulus, specified at a reference mean } \\
\text { effective confining pressure refPress of } \mathrm{p}_{\mathrm{r} \text {. }}\end{array}$ \\
\hline refBulkModu & $\begin{array}{l}\text { Reference bulk modulus, specified at a reference mean effective confining pressure } \\
\text { refPress of } p_{r}^{\prime} \text {. }\end{array}$ \\
\hline frictionAng & Friction angle at peak shear strength, in degrees. \\
\hline peakShearStra & $\begin{array}{l}\text { An octahedral shear strain at which the maximum shear strength is reached, specified } \\
\text { at a reference mean effective confining pressure refPress of } \mathrm{p}_{\mathrm{r}} \text {. }\end{array}$ \\
\hline refPress & Reference mean effective confining pressure at which $\mathrm{G}_{\mathrm{r}}, \mathrm{B}_{\mathrm{r}}$, and $\Upsilon_{\max }$ are defined. \\
\hline pressDependCoe & $\begin{array}{l}\text { A positive constant defining variations of } \mathrm{G} \text { and } \mathrm{B} \text { as a function of instantaneous } \\
\text { effective confinement } \mathrm{p} \text {. }\end{array}$ \\
\hline PTAng & Phase transformation angle, in degrees. \\
\hline Contrac & $\begin{array}{l}\text { A non-negative constant defining the rate of shear-induced volume decrease } \\
\text { (contraction) or pore pressure buildup. A larger value corresponds to faster } \\
\text { contraction rate. }\end{array}$ \\
\hline dilat1, dilat 2 & $\begin{array}{l}\text { Non-negative constants defining the rate of shear-induced volume increase (dilation). } \\
\text { Larger values correspond to stronger dilation rate. }\end{array}$ \\
\hline $\begin{array}{l}\text { Liquefac } 1 \text {, liquefac } 2 \text {, } \\
\text { liquefac } 3\end{array}$ & $\begin{array}{l}\text { Parameters controlling the mechanism of liquefaction-induced perfectly plastic shear } \\
\text { strain accumulation. }\end{array}$ \\
\hline $\mathrm{e}$ & Initial void ratio. \\
\hline
\end{tabular}

$[C]=\alpha[M]+\beta[K]$

In 2004, Park and Hashash explained how to use the Rayleigh formulation in site response analysis. $\alpha$ and $\beta$ are calculated from two frequencies of significant natural modes i and j using Eq. 25.

$$
\left[\begin{array}{l}
\xi_{i} \\
\xi_{j}
\end{array}\right]=\frac{1}{4 \pi}\left[\begin{array}{ll}
\frac{1}{f_{i}} & f_{i} \\
\frac{1}{f_{j}} & f_{j}
\end{array}\right]\left\{\begin{array}{l}
\alpha \\
\beta
\end{array}\right\}
$$

where $f_{i}$ and $f_{j}$ are the significant frequencies. $\xi_{i}$ and $\xi_{j}$ are the damping ratios of $f_{i}$ and $f_{j}$, respectively.

In 2008, Stewart and Kwok recommended selecting the first frequency as the natural frequency of the site and the second frequency as approximately five times that of $f_{i}$. Natural frequency is commonly calculated by the following equation:

$$
f_{n}=\frac{V_{s}}{4 H}
$$

In the current study, the significant frequencies were determined from the recommendation of Stewart and Kwok (2008). The results of the linear time domain were compared with those of the frequency domain for a specific site in DEEPSOIL. Two significant frequencies were selected based on minimizing the difference between the results of the time domain and the frequency domain. The full Rayleigh damping was selected and its parameters were calculated using the two significant frequencies mentioned above.
Rayleigh damping was modeled in DEEPSOIL based on the frequency-independent viscous damping proposed by Philips and Hashash (2009).

\section{RESULTS AND DISCUSSION \\ 4.1. LINEAR SITE RESPONSE ANALYSIS KOBE EARTHQUAKE:}

The 1D site response was modeled in DEEPSOIL software. In this software, the linear timedomain approach was selected for modeling a $20 \mathrm{~m}$ soil profile with an elastic half-space. The shear wave velocities of the soil layer and the unbounded domain were selected as $250 \mathrm{~m} / \mathrm{s}$ and $750 \mathrm{~m} / \mathrm{s}$ respectively.

The elastic modulus and Poisson's ratio were used in the SBFEM to model the soil behavior. The elastic modulus of the soil layer was determined based on Eq. 27. The Poisson's ratios for the soil layers and the bedrock were selected as 0.3 and 0.25 , respectively.

$G=\frac{E}{2(1+\mu)}=\rho V_{s}^{2}$

Three subdomains were selected for modeling the bounded domain, while the unbounded domain was modeled in one subdomain. The subdomains of the model are illustrated in Figure 5.

In the bounded domain, two subdomains were designated for modeling the soil column. Another subdomain was considered to model the base rock. The seismic force was applied at the soil and rock interface. The bedrock material was assigned to domain 3, line 8, line 9, and line 10. Each subdomain consisted of a center point (c) and several lines (L). $\mathrm{C}_{1}$ to $\mathrm{C}_{3}$ were the centers of the near-field subdomains. Point $\mathrm{C}_{4}$ was the center of the far-field subdomain. 


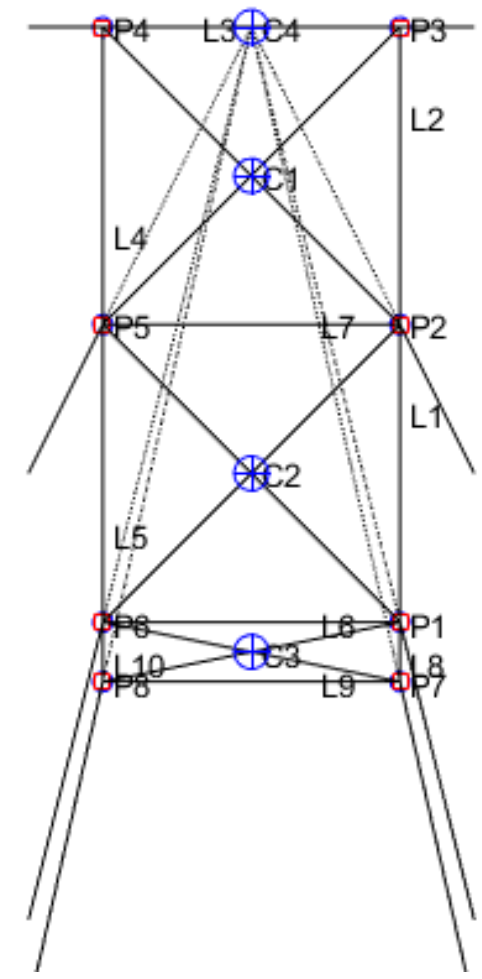

Fig. 5 The subdomains of the site response analysis.
In Figure 6, the displacement results at the top of the model (on the ground surface) obtained from the scaled boundary finite element are presented for the Kobe earthquake.

In Figure 6, $\mathrm{M}$ is the continued fraction order. The effect of the continued fraction order of the unbounded domain on the results is shown in Figure 6. As shown, after three fractions, the results were converged on the same line and increasing the fraction order did not have any effect on displacement on the top of the surface.

The spectral accelerations with a five percent damping ratio obtained using DEEPSOIL and the SBFEM are plotted in Figure 7. As seen, the maximum displacement in the surface of the soil layer calculated using the SBFEM has a good agreement with the result obtained from the linear model developed in DEEPSOIL.

The Fourier amplitudes of ground surface responses for DEEPSOIL and the SBFEM were also calculated and shown in Figure 8.

The relative difference between two types of analysis is usually calculated based on Eq. 28 .

$$
R(T)=\ln \left(S_{a}(T)\right)_{\text {Deepsoil }}-\ln \left(S_{a}(T)\right)_{\text {SBFEM }}
$$

where $R(T)$ is the residual, $\ln \left(S_{a}(T)\right)_{\text {Deepsoil }}$ is the natural log of spectral acceleration (using the DEEPSOIL modeling), and $\ln \left(S_{a}(T)\right)_{S B F E M}$ is the natural $\log$ of spectral acceleration (using the SBFEM modeling) at period $\mathrm{T}$. $\mathrm{R}(\mathrm{T})$ for linear analysis is shown in Figure 9.

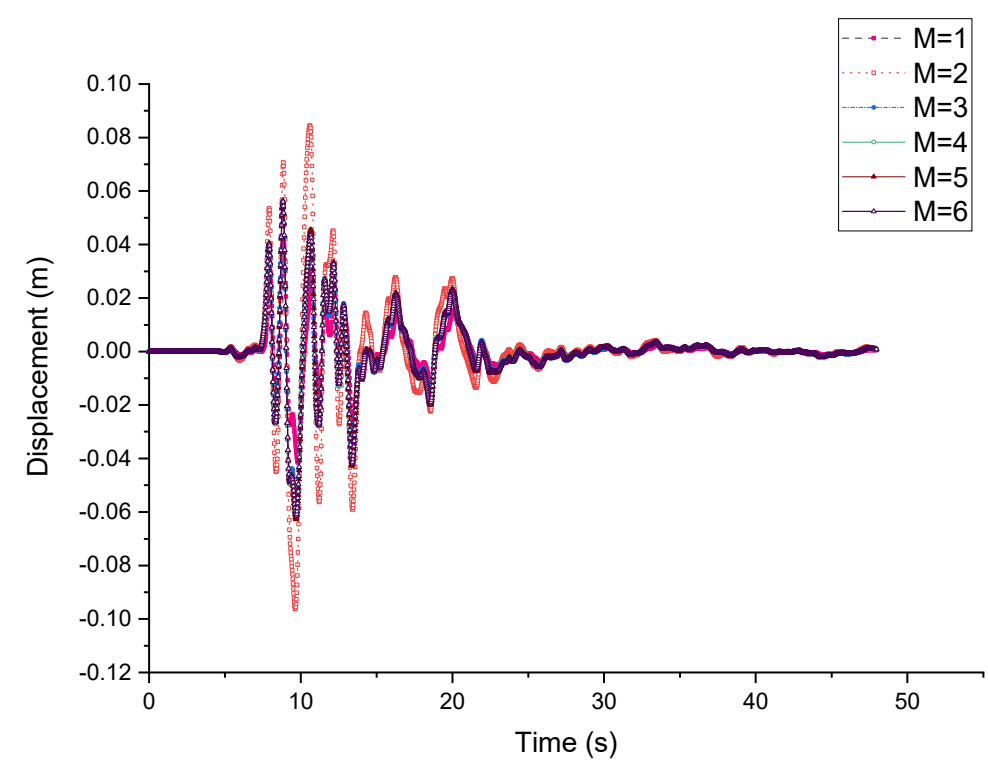

Fig. 6 The horizontal displacement of the ground surface (Kobe earthquake). 


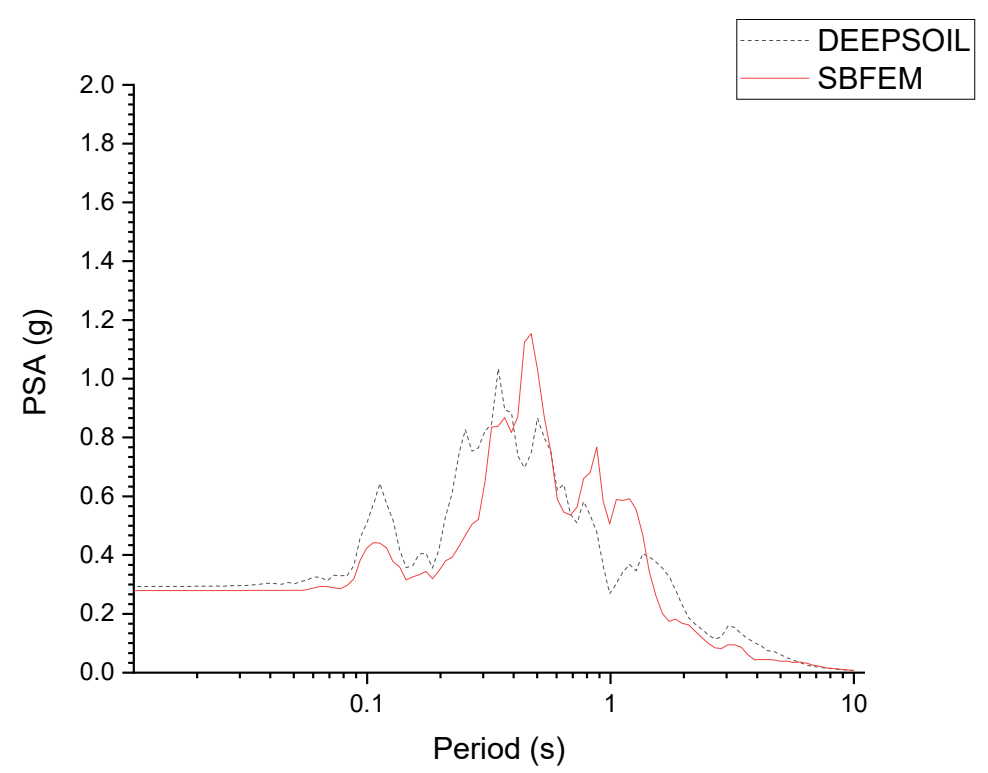

Fig. 7 The spectral accelerations obtained with DEEPSOIL and the SBFEM (linear analyses).

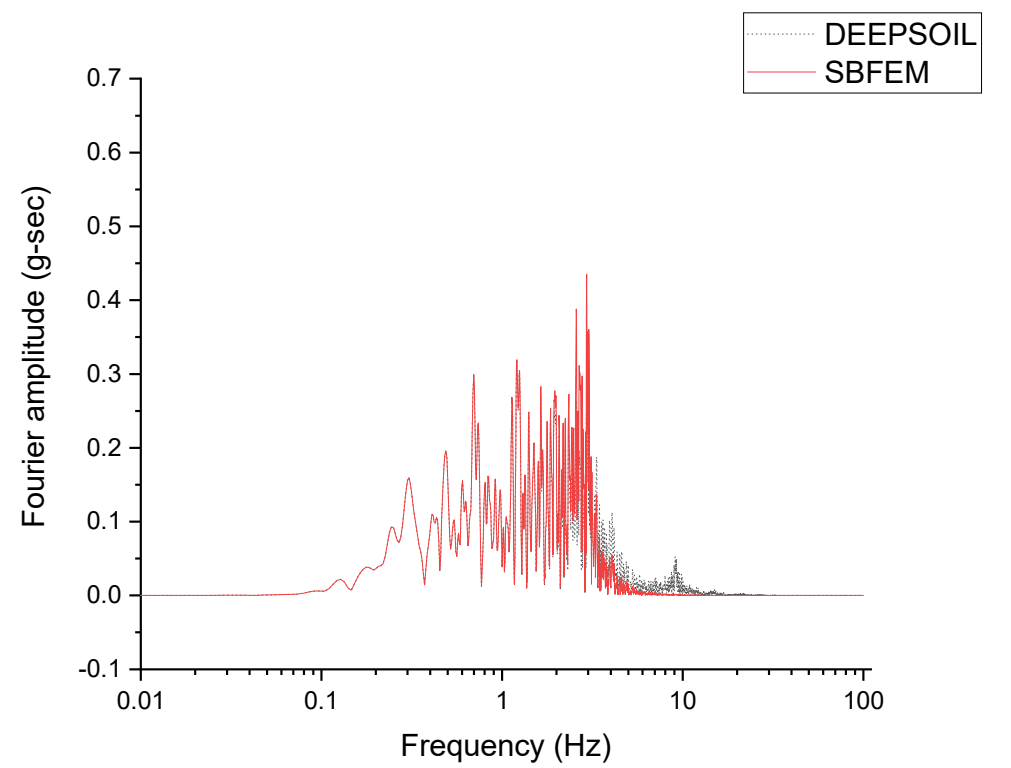

Fig. 8 The Fourier amplitudes (linear analyses).

As seen in Figure 9, compared to DEEPSOIL, the SBFEM overestimates spectral acceleration in low periods and underestimates it in high periods. A little time-shifting and period lengthening were observed in the 2D SBFEM/FEM site response analysis (Fig. 9) that are similar to the period lengthening in the soilstructure interaction analysis. Period lengthening has a major effect on the behavior of the soil-structure interaction in both linear and nonlinear modeling.

The effects of three other large earthquakes on the soil column were also modeled in the SBFEM and the results are presented below.

As shown in Figures 10-12, the seismic horizontal displacement of the ground surface for all the shaking events was matched on one curve for $\mathrm{M}>3$. For $\mathrm{M}<3$, displacement was overestimated.
The displacement at the interface points (the displacements of $\mathrm{P}_{4}$ and $\mathrm{P}_{3}$ in Fig. 5) is a significant parameter in the magnitude of the interaction force. The displacement on both sides of the model for the Kobe earthquake was determined and illustrated in Figure 13.

As shown in Figure 13, in the SBFEM, the displacement is equal at both sides of the model. This condition occurs in OpenSees and in the free-field modeling of the absorbing boundary. The degrees of freedom of both nodes are equalized and the finite element solution is prepared based on the boundary conditions of the two corresponding nodes. Due to the symmetry in the model, the displacements at both sides of the model were equal. Therefore, it is reasonable to conclude that the free-field boundary 


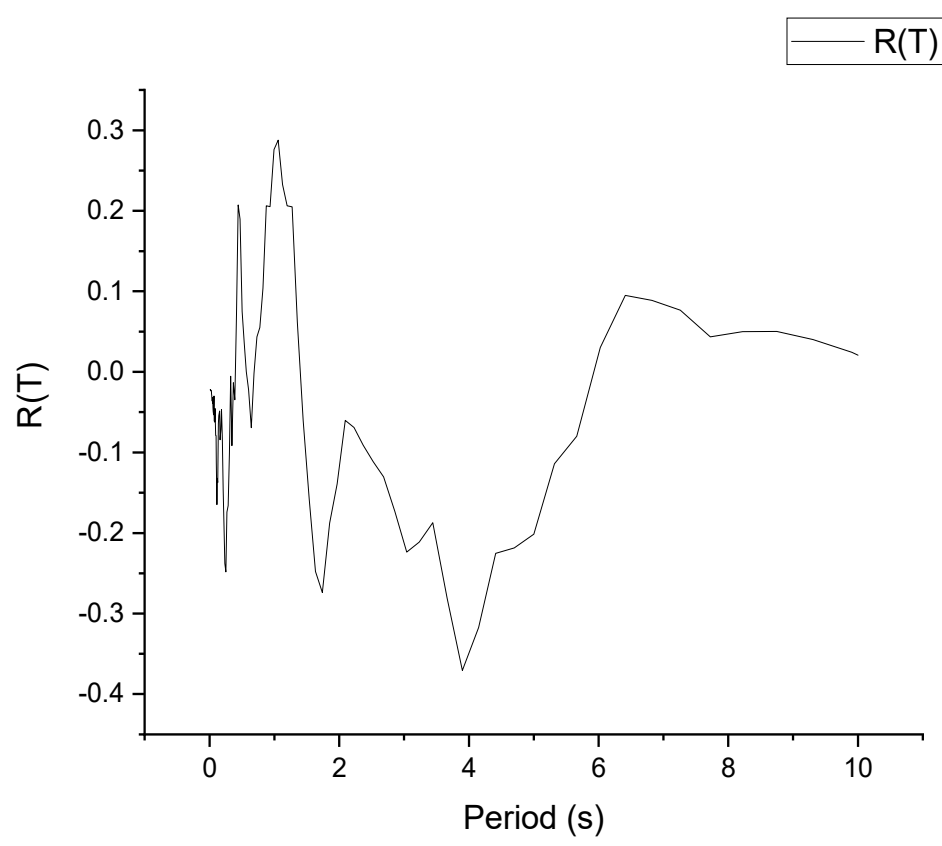

Fig. 9 The relative difference between the results of DEEPSOIL and SBFEM (linear analyses).

\section{Imperial Valley Earthquake:}

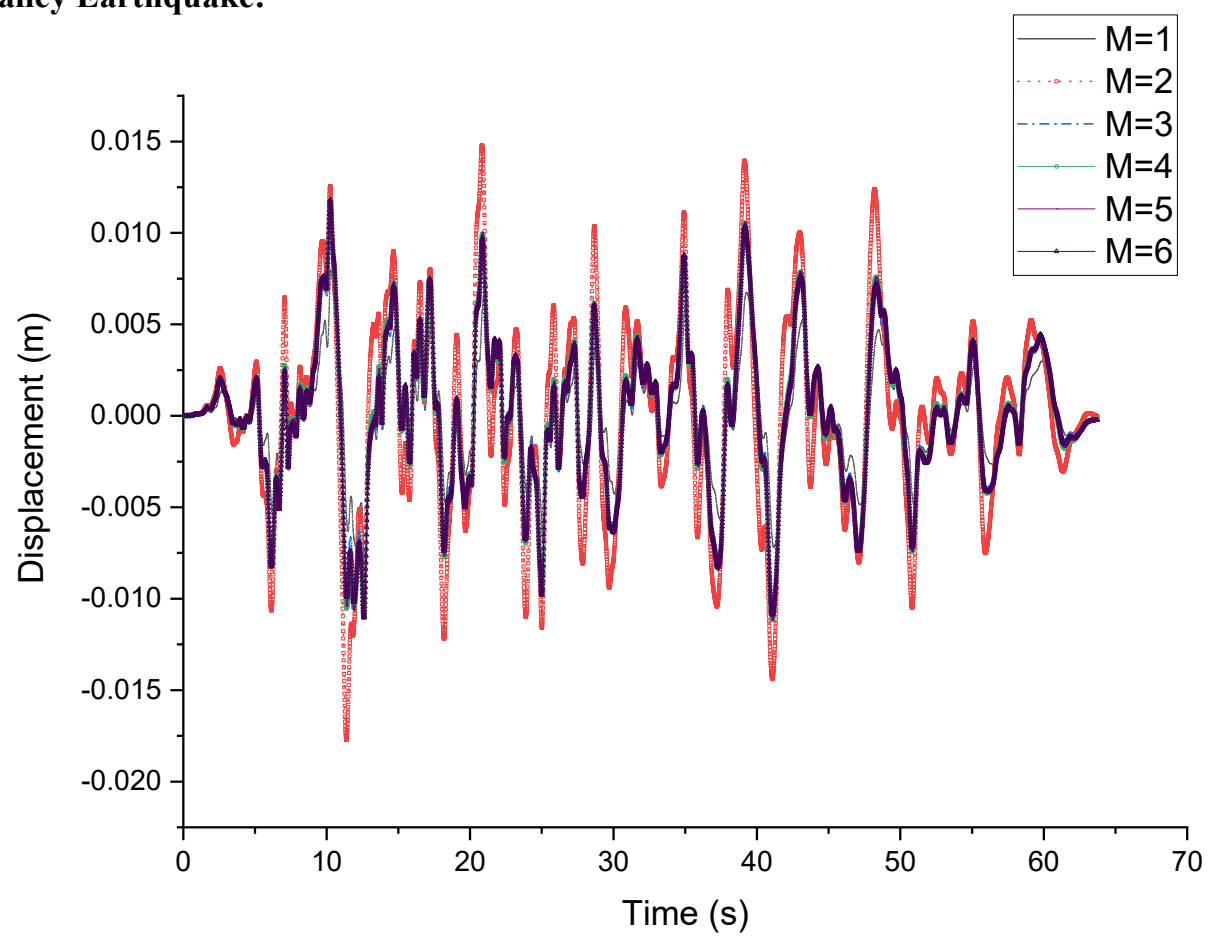

Fig. 10 The horizontal displacement of the ground surface (the Imperial Valley earthquake).

conditions are promising in a model that has a symmetric geometry.

\subsection{NONLINEAR SITE RESPONSE ANALYSES}

The nonlinear site response analyses of a $20 \mathrm{~m}$ soil layer on the rock were modeled using DEEPSOIL. All the inputs have been described in the previous section. In the coupled SBFEM/FEM model, the bounded domain was modeled using a 2D FEM framework with 220 quadratic elements and 299 nodes as shown in Figure 14. The size of the mesh was selected smaller than wavelength/15. Truncated line elements were generated for modeling the interface between the far-field and the near-field. The DOFs of the truncated line element nodes were equalized to the outer nodes of the near-field mesh. The unbounded 


\section{Chichi Earthquake:}

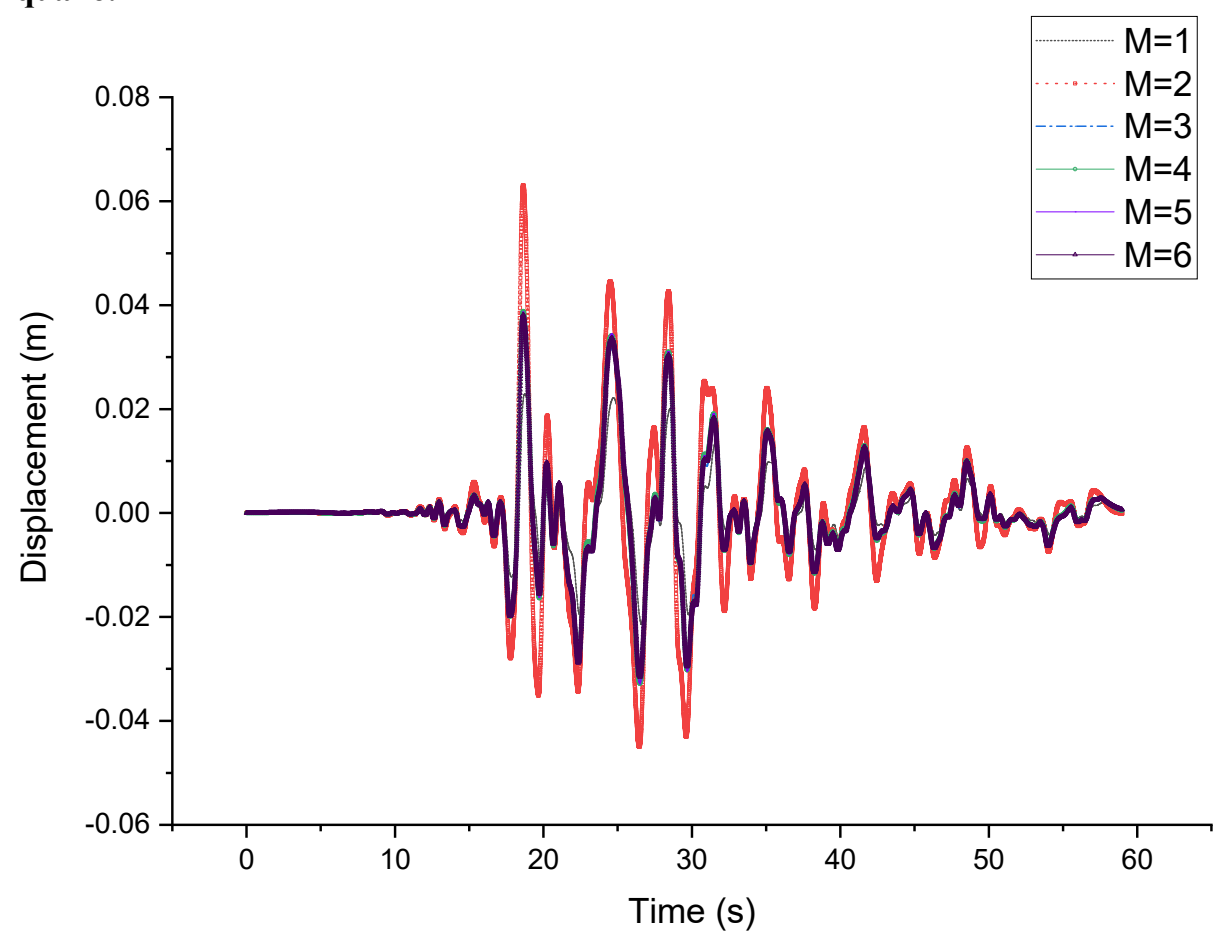

Fig. 11 The horizontal displacement of the ground surface (the Chichi earthquake).

\section{Kocaeli Earthquake:}

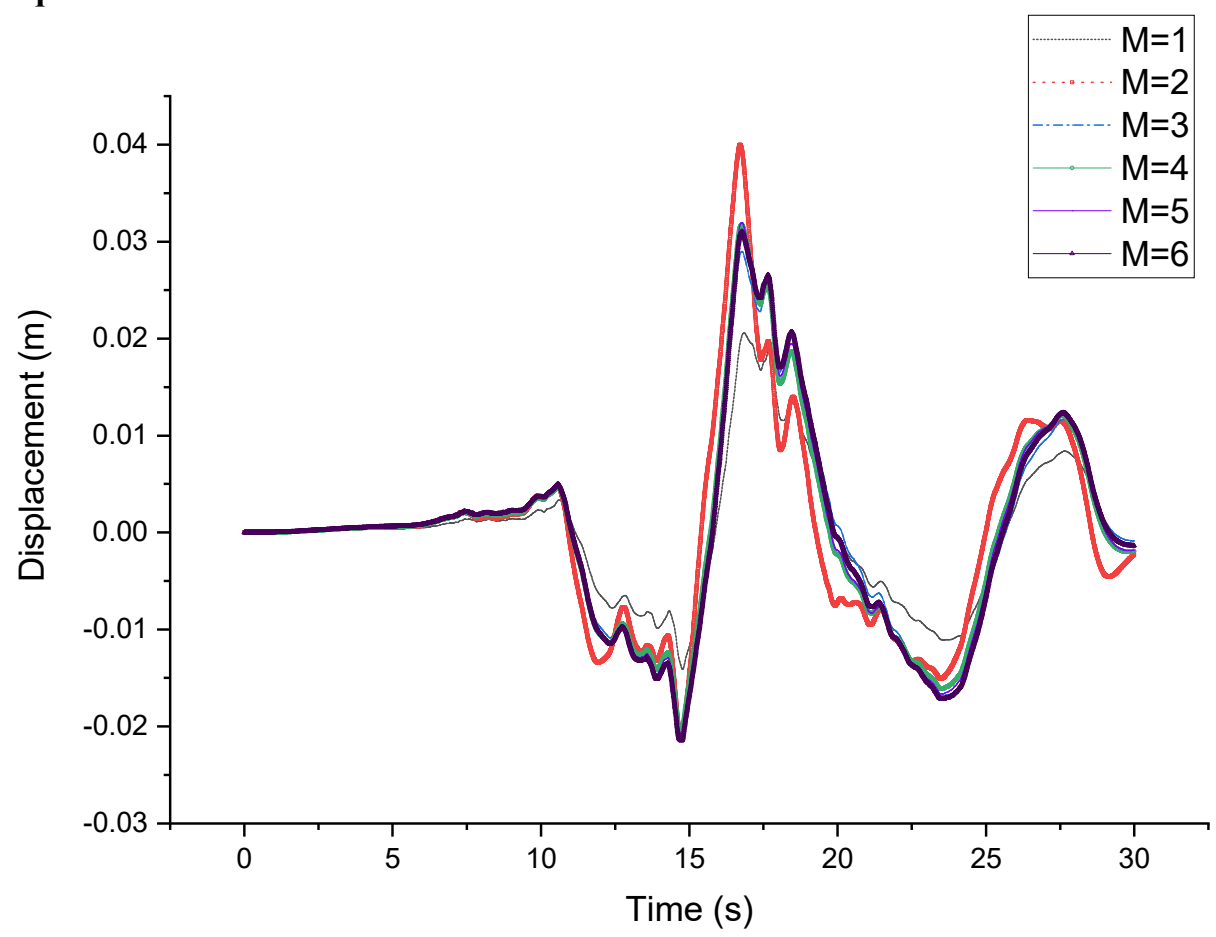

Fig. 12 The horizontal displacement of the ground surface (the Kocaeli earthquake).

domain equations (Eq. 21) were solved in MATLAB for the desired geometry (Fig. 14). The matrices of high-order asymptotic boundary equations were obtained only once. The sequence for the determination of the matrices has been mentioned in section 2. The MATLAB scripts in Eq. 21 were implemented in the OpenSees platform using a new element generated in OpenSees. The displacements and velocities of the truncated line element nodes were used in this element and the interaction force was calculated in each time step and was applied to the nodes. 


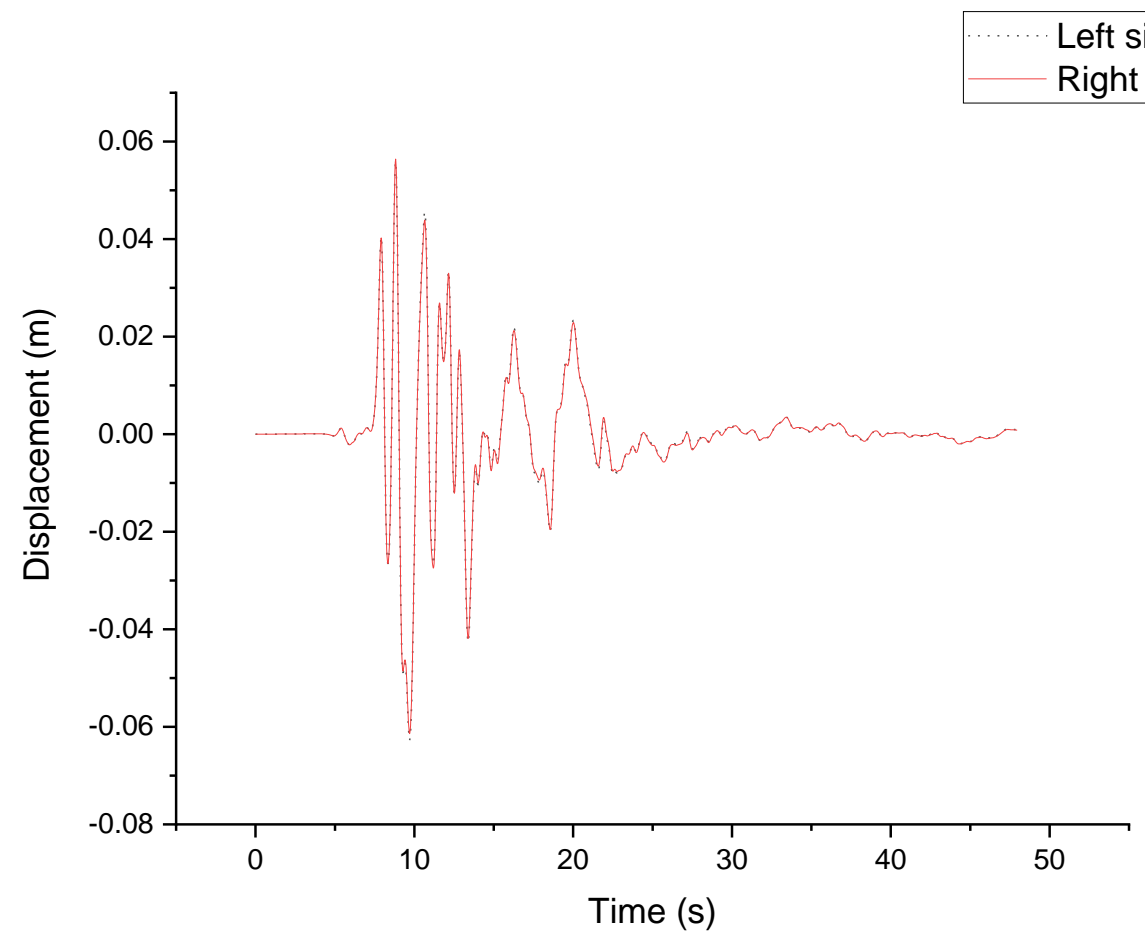

Fig. 13 The displacement of both sides of the model (the Kobe earthquake).

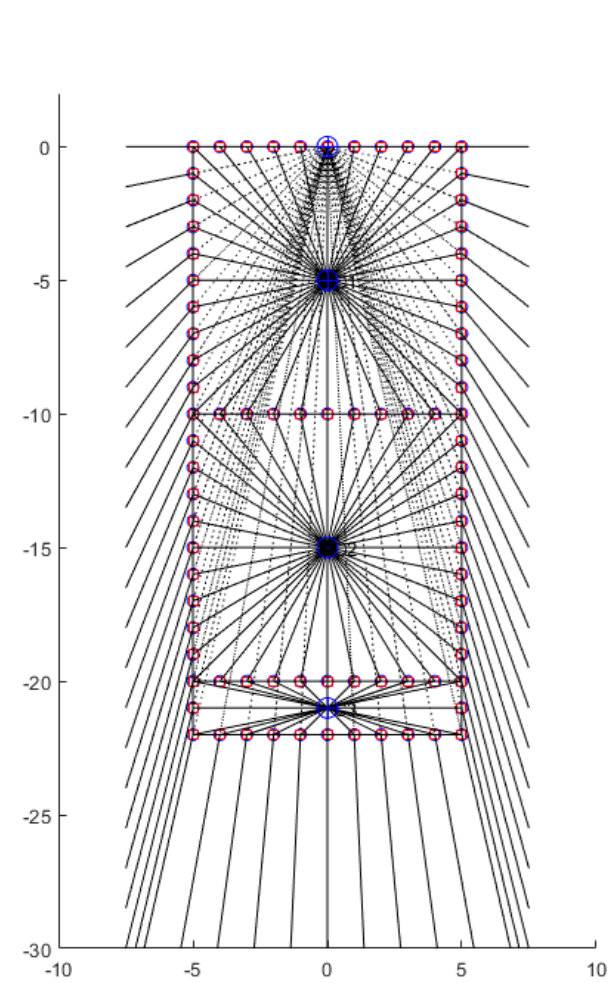

a

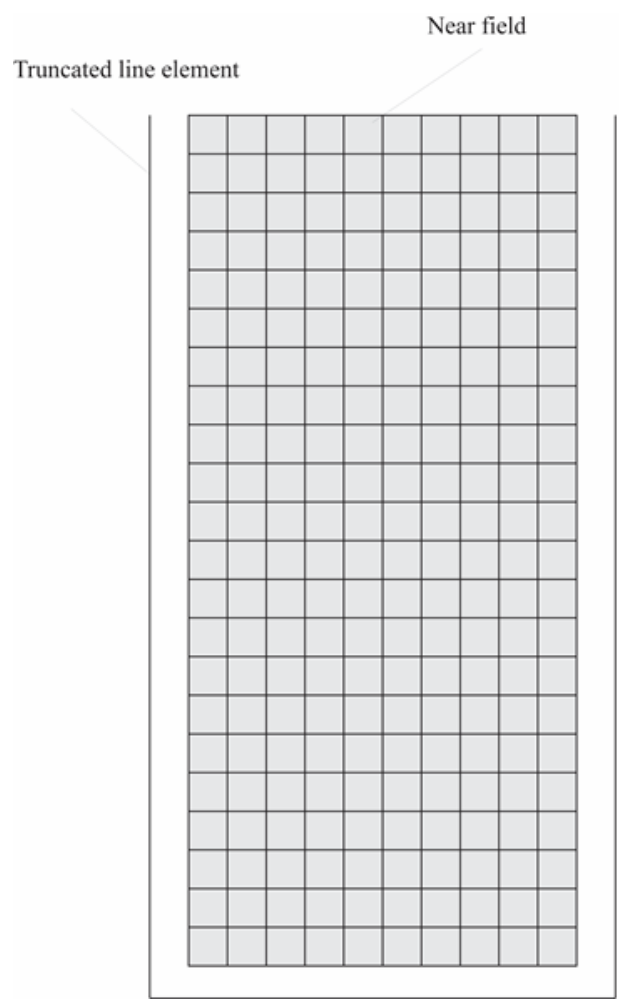

b

Fig. 14 The bounded and unbounded domains in the nonlinear analyses a) the SBFEM model, b) the bounded domain. 


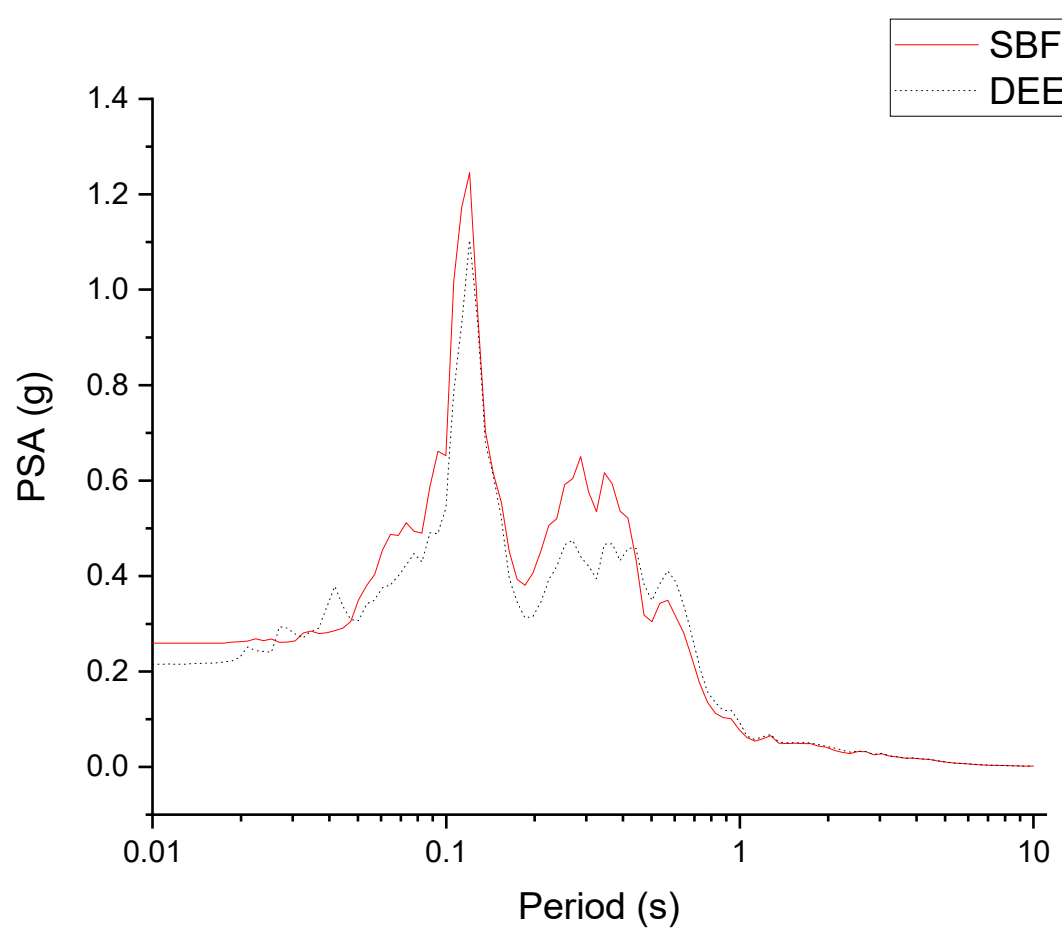

Fig. 15 The spectral accelerations obtained from DEEPSOIL and the SBFEM (nonlinear analyses).

Table 4 Nonlinear parameters of soil.

\begin{tabular}{ll}
\hline Parameter & Description \\
\hline Rho & 1.9 ton $/ \mathrm{m}^{3}$ \\
refShearModul & $75000 \mathrm{kPa}$ \\
refBulkModu & $200000 \mathrm{kPa}$ \\
frictionAng & 33 degree \\
peakShearStra & 0.1 \\
refPress & $80 \mathrm{kPa}$ \\
pressDependCoe & 0.5 \\
PTAng & 27 degree \\
Contrac & 0.07 \\
dilat1, dilat 2 & $0.4,2$ \\
liquefac 1, & $10 \mathrm{kPa}$ \\
liquefac 2, & 0.01 \\
liquefac 3 & \\
e & 1 \\
\hline
\end{tabular}

Rayleigh damping was applied to the whole bounded domain and the Kobe ground motion record was employed as a seismic input motion in all the nonlinear analyses. The soil model parameters are tabulated in Table 4.

In this section, the results of the nonlinear site response analyses with two modeling methods are presented. The spectral accelerations for DEEPSOIL and the SBFEM/FEM with a $5 \%$ damping ratio are plotted in Figure 15.

The Fourier amplitudes of the ground surface for the nonlinear analyses are presented in Figure 16.
$R(T)$ is shown in Figure 17 for the nonlinear analyses.

As seen, in nonlinear modeling, $R(T)$ is a very small number at high periods, whereas it has a reasonable value in low periods. In low periods, the SBFEM underestimated the spectral acceleration compared with DEEPSOIL modeling. The location of PSA obtained with the nonlinear SBFEM/FEM approach was in good agreement with the prediction of DEEPSOIL especially in high period ranges.

\section{CONCLUSIONS}

In this study, a nonlinear time-domain site response analysis was presented using the SBFEM/FEM coupled method. The bounded domain was modeled by utilizing the OpenSees software. The far-field unbounded domain was modeled by an improved high-order transmitting boundary. The nonlinear damping model and the behavior of the soil were taken into consideration for modeling the soil media in both the SBFEM/FEM and DEEPSOIL software. The damping model in the SBFEM/FEM was determined based on two significant frequencies.

The results of the coupled model were compared with those of the DEEPSOIL nonlinear analysis. The obtained residual parameter was less than 0.5 , demonstrating the accuracy of the coupling method. Similar conclusions were drawn in the linear timedomain analyses.

This modeling method can be used in the nonlinear time-domain soil-structure interaction analysis which is the topic of our next paper. 


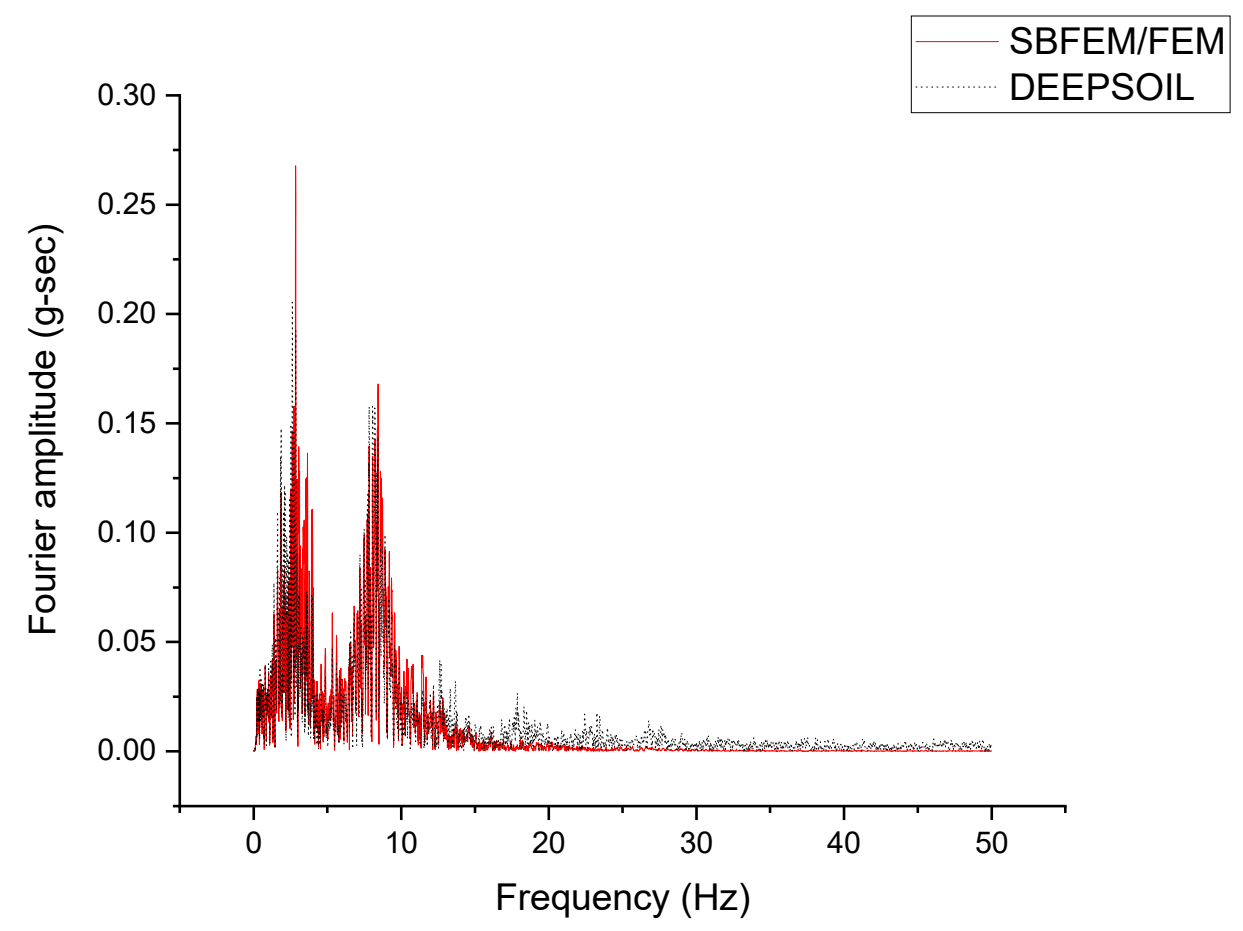

Fig. 16 The Fourier amplitudes (nonlinear analyses).

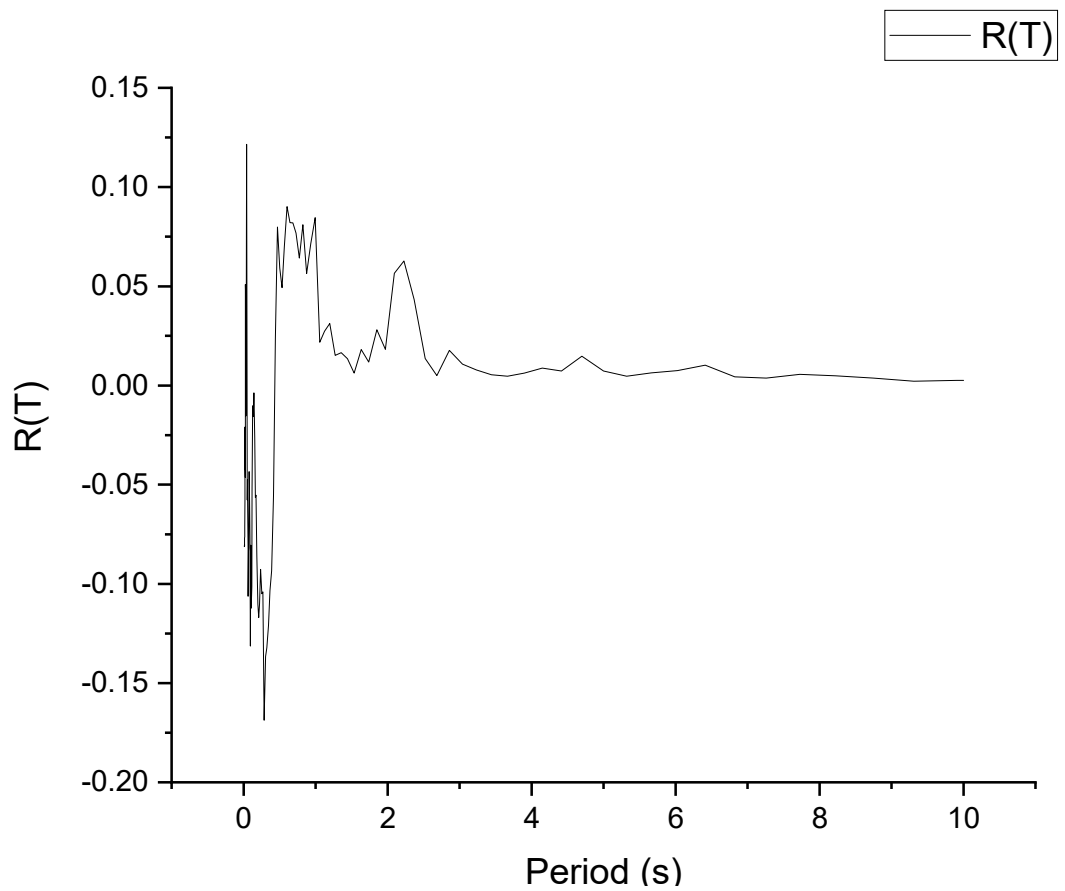

Fig. 17 The relative difference between the results of DEEPSOIL and the SBFEM (nonlinear analyses).

\section{REFERENCES}

Afacan, K.B., Yniesta, S., Shafiee, A., Stewart, J.P. and Brandenberg, S.J.: 2019, Total stress analysis of soft clay ground response in centrifuge models. J. Geotech. Geoenviron. Eng., 145, 10, 04019061.

DOI: 10.1061/(ASCE)GT.1943-5606.0002115

Ancheta, T.D., Darragh, R.B., Stewart, J.P., Seyhan, E., Silva, W.J., Chiou, B.S., and Donahue J.L.: 2014,
NGA-West2 database. Earthq. Spectra, 30, 3, 9891005. DOI: 10.1193/070913EQS197M

Arslan, H. and Siyahi, B.: 2006, A comparative study on linear and nonlinear site response analysis. Environ. Geol., 50, 8, 1193-1200.

DOI: $10.1007 / \mathrm{s} 00254-006-0291-4$

Bazyar, M.H. and Song, C.: 2008, A continued-fractionbased high-order transmitting boundary for wave propagation in unbounded domains of arbitrary 
geometry. Int. J. Numer. Meth. Eng., 74, 2, 209-237. DOI: $10.1002 / \mathrm{nme} .2147$

Bazyar, M.H. and Song, C.: 2017, Analysis of transient wave scattering and its applications to site response analysis using the scaled boundary finite-element method. Soil Dyn. Earthq. Eng., 98, 191-205. DOI: $10.1016 /$ j.soildyn.2017.04.010

Birk, C., Prempramote, S. and Song, C.: 2012, An improved continued-fraction-based high-order transmitting boundary for time-domain analyses in unbounded domains. Int. J. Numer. Meth. Eng., 89, 3, 269-298. DOI: $10.1002 / \mathrm{nme} .3238$

Choi, Y. and Stewart, J.P.: 2005, Nonlinear site amplification as function of $30 \mathrm{~m}$ shear wave velocity. Earthq. Spectra, 21, 1, 1-30. DOI: 10.1193/1.1856535

Darendeli, M.B.: 2001, Development of a new family of normalized modulus reduction and material damping curves. Ph.D Dissertation, The University of Texas at Austin.

Elgamal, A., Lai, T., Yang, Z. and He, L.: 2001, Dynamic soil properties, seismic downhole arrays and applications in practice. International Conferences on Recent Advances in Geotechnical Earthquake Engineering and Soil Dynamics, 6.

https://scholarsmine.mst.edu/icrageesd/04icrageesd/session13/6

Field, E.H. and Petersen, M.D.: 2000, A test of various siteeffect parameterizations in probabilistic seismic hazard analyses of southern California. Bull. Seismol. Soc. Am., 90, 6B, S222-S244. DOI: $10.1785 / 0120000502$

Harmsen, S.C.: 1997, Determination of site amplification in the Los Angeles urban area from inversion of strongmotion records. Bull. Seismol. Soc. Am., 87, 4, 866887.

Hashash, Y.M.A, Phillips, C. and Groholski, D.R.: 2010, Recent advances in non-linear site response analysis. International Conferences on Recent Advances in Geotechnical Earthquake Engineering and Soil Dynamics, 8.

https://scholarsmine.mst.edu/icrageesd/05icrageesd/session $12 / 8$

Hashash, Y.M.A. and Moon, S.W.: 2011, Site amplification factors for deep deposits and their application in seismic hazard analysis for central U.S. Final Report, USGS/NERP Grant: G09AP00123. US Geological Survey.

Hashash, Y., Dashti, S., Romero, M., Ghayoomi, M. and Musgrove, M.: 2015, Evaluation of 1-D seismic site response modeling of sand using centrifuge experiments. Soil Dyn. Earthq. Eng., 78, 19-31. DOI: 10.1016/j.soildyn.2015.07.003

Hashash, Y.M.A., Musgrove, M.I., Harmon, J.A., Okan, I., Groholski, D.R., Phillips, C.A. and Park, D.: 2017, DEEPSOIL 7.0, user manual. University of Illinois at Urbana-Champaign.

Joyner, W.B. and Chen, A.T.: 1975, Calculation of nonlinear ground response in earthquakes. Bull. Seismol. Soc. Am., 65, 5, 1315-1336.

Joyner, W.B. and Boore, D.M.: 2000, Recent developments in earthquake ground motion estimation. Proceedings of the Sixth International Conference on Seismic Zonation, 12-15.

Kaklamanos, J., Baise, L.G., Thompson, E.M. and Dorfmann, L.: 2015, Comparison of 1D linear, equivalent-linear, and nonlinear site response models at six KiK-net validation sites. Soil Dyn. Earthq. Eng., 69, 207-219. DOI: 10.1016/j.soildyn.2014.10.016
Kim, B., Hashash, Y.M.A., Stewart, J.P., Rathje, E.M., Harmon, J.A., Musgrove, M.I., and Silva, W.J.: 2016, Relative differences between nonlinear and equivalent-linear 1-D site response analyses. Earthq. Spectra, 32, 3, 1845-1865. DOI: $10.1193 / 051215 E Q S 068 \mathrm{M}$

Kwok, A.O., Stewart, J.P., Hashash, Y.M.A., Matasovic, N., Pyke, R., Wang, Z. and Yang, Z.: 2007, Use of exact solutions of wave propagation problems to guide implementation of nonlinear seismic ground response analysis procedures. J. Geotech. Geoenviron. Eng., $133,11,1385-1398$

DOI: 10.1061/(ASCE)1090-0241(2007)133:11(1385)

Kwok, A.O., Stewart, J.P. and Hashash, Y.M.A: 2008, Nonlinear ground-response analysis of Turkey flat shallow stiff-soil site to strong ground motion. Bull. Seismol. Soc. Am., 98, 1, 331-343. DOI: $10.1785 / 0120070009$

Lopez-Caballero, F., Razavi, A.M.F. and Modaressi, H.: 2007, Nonlinear numerical method for earthquake site response analysis I-elastoplastic cyclic model and parameter identification strategy. Bull. Earthq. Eng., 5, 3, 303-323. DOI: 10.1007/s10518-007-9032-7

Lysmer, J. and Kuhlemeyer, R.L.: 1969, Finite dynamic model for infinite media. J. Eng. Mech. Div., 95, 4, 859-878.

Mazzoni, S., McKenna, F., Scott, M.H and Fenves, G.L.: 2006, OpenSees command language manual. Pacific Earthquake Engineering Research (PEER) Center, 264.

Mirshekari, M. and Ghayoomi, M.: 2015, Simplified equivalent linear and nonlinear site response analysis of partially saturated soil layers. IFCEE 2015, 21312140. DOI: $10.1061 / 9780784479087.197$

Park, D. and Hashash, Y.M.A.: 2004, Soil damping formulation in nonlinear time domain site response analysis. J. Earthq. Eng., 8, 02, 249-274. DOI: $10.1142 / \mathrm{S} 1363246904001420$

Phillips, C. and Hashash, Y.M.A.: 2009, Damping formulation for nonlinear 1D site response analyses. Soil Dyn. Earthq. Eng., 29, 7, 1143-1158. DOI: $10.1016 /$ j.soildyn.2009.01.004

Pruiksma, J.P.: 2016, Nonlinear and equivalent linear site response analysis for the Groningen area. TNO Report, R10460. Delft, the Netherlands.

Rayleigh, J.W.S. and Lindsay, R.B.: 1945, The theory of sound, I and 2, $1^{\text {st }}$ American ed., Dover, New York, 130-132.

Régnier, J., Bonilla, L.F., Bard, P.Y., Bertrand, E., Hollender, F., Kawase, H., ..., and Verruci, L.: 2018. PRENOLIN: International benchmark on 1D nonlinear: Site-response analysis-validation phase exercise. Bull. Seismol. Soc. Am., 108, 2, 876-900. DOI: $10.1785 / 0120170210$

Rong, M., Wang, Z., Woolery, E.W., Lyu, Y., Li, X. and Li, S.: 2016. Nonlinear site response from the strong ground-motion recordings in western China. Soil Dyn. Earthq. Eng., 82, 99-110. DOI: $10.1016 /$ j.soildyn.2015.12.001

Seed, H.B. and Idriss, I.M.: 1969, Influence of soil conditions on ground motions during earthquakes. J. Soil Mech. Found. Div., 95, 1, 99-138.

Seyhan, E. and Stewart, J.P.: 2014, Semi-empirical nonlinear site amplification from NGA-West2 data and simulations. Earthq. Spectra, 30, 3, 1241-1256. DOI: $10.1193 / 063013 \mathrm{EQS181M}$ 
Song, C.: 2006, Dynamic analysis of unbounded domains by a reduced set of base functions. Comput. Methods Appl. Mech. Eng., 195, 33-36, 4075-4094.

DOI: 10.1016/j.cma.2005.07.011

Song, C.: 2018, The scaled boundary finite element method: Introduction to theory and implementation. John Wiley \& Sons.

Song, C. and Bazyar, M.H.: 2007, A boundary condition in Padé series for frequency-domain solution of wave propagation in unbounded domains. Int. J. Numer. Meth. Eng., 69, 11, 2330-2358.

DOI: $10.1002 / \mathrm{nme} .1852$

Song, C. and Wolf, J.P.: 1998, The scaled boundary finiteelement method: Future developments. Proc. IUTAM Symposium. Fluid Mech. its Appl., 49, 275-284.

Stewart, J.P. and Kwok, A.O.: 2008, Nonlinear seismic ground response analysis: Code usage protocols and verification against vertical array data. Proc. Geotechnical Earthquake Engineering and Soil Dynamics IV, 1-24. DOI: 10.1061/40975(318)1

Tsai, C.C. and Hashash, Y.M.A.: 2009, Learning of dynamic soil behavior from downhole arrays. J. Geotech. Geoenviron. Eng., 135, 6, 745-757.

DOI: 10.1061/(ASCE)GT.1943-5606.0000050

Tsai, C.C., Park, D. and Chen, C.W.: 2014, Selection of the optimal frequencies of viscous damping formulation in nonlinear time-domain site response analysis. Soil Dyn. Earthq. Eng., 67, 353-358.

DOI: $10.1016 /$ j.soildyn.2014.10.026

Wolf, J.P.: 2003, The scaled boundary finite element method. John Wiley \& Sons.

Yee, E., Stewart, J.P. and Tokimatsu, K.: 2013, Elastic and large-strain nonlinear seismic site response from analysis of vertical array recordings. J. Geotech. Geoenviron. Eng., 139, 10, 1789-1801.

DOI: 10.1061/(ASCE)GT.1943-5606.0000900 